\title{
Micropropagation of Four Coffee Cultivars (Coffea arabica L.) from Yemen through Shoot Tip Culture
}

\author{
Ameen, A. AL-Azab ${ }^{1}$; Sanad, S. M. Habib ${ }^{2}$; Mohammed, A. Hussein ${ }^{3}$ and Fadia El-Sherif ${ }^{4 *}$ \\ ${ }^{1,2,4}$ Horticulture Dept., Faculty of Agriculture, Suez Canal University, 41522, Ismailia, Egypt \\ ${ }^{3}$ Botany Dept., Faculty of Agriculture, Suez Canal University, 41522, Ismailia, Egypt. \\ * Department of Biological Sciences, Faculty of Science, King Faisal University, \\ P.O. Box. 380, Al-Ahsaa 31982, Kingdom of Saudi Arabia.
}

\section{Received: $20 / 11 / 2015$}

\begin{abstract}
The aim of this study is to optimize in vitro multiplication of Coffea arabica cvs 'Benan',' Burai',' Odayni' and 'Odayni- Bayat'. Seeds germinated on modified quarter MS medium with $1.0 \mathrm{~g} \mathrm{1^{-1 }}$ activated charcoal gave the highest germination percentage of the four cultivars under study. BA at $8.0 \mathrm{mgl}^{-1}$ was the most effective concentration with an average of 14 shoots per explant, resulting in better and morphologically superior microshoots in 'Benan' cv. However 'Burai' ,'Odayni' and 'Odayni- Bayat' cultivars, showed the highest number of shoots when they were grown on MS medium supplemented with BA at 4.0, 2.0 and $6.0 \mathrm{mgl}^{-1}$ respectively. Root formation was $100 \%$ in response to the application of IAA, IBA and /or NAA to the culture media for the four cultivars. Half MS supplemented with NAA at 2.0, 1.0 and $3.0 \mathrm{mgl}^{-1}$ was found to be a suitable medium for root induction in excised micro-shoots, of 'Odayni', 'Burai' and 'Odayni-Bayat' cultivars, respectively. The plantlets were successfully acclimatized in the greenhouse; consequently the survived plants reached $100 \%$.
\end{abstract}

Keywords: In vitro, Benzyladenine, Coffea arabica. 'Benan', 'Burai', 'Odayni' and Odayni-Bayat, micropropagation,

\section{INTRODUCTION}

Coffee belongs to the family Rubiacea. The genus Coffee includes at least 64 species grouped into four sections (Carvalho and Monaco, 1969). Commercially, only two out of more than 100 coffee species are cultivated, the $C$. arabica (Arabica) and C. canephora (Robusta) (Pierson et al., 1983; Juma et al., 1994; Carneiro, 1997). The most important species is $C$. arabica. In Yemen, there are many coffee cultivars and they are named according to their place of origin (Robinson and Brian, 1993). There are many countries which depend in their economy on the coffee exportation (Juma et al., 1994). Quality beverage is produced from $C$. arabica which is cultivated at higher altitudes. This species represent $70 \%$ of the commercial coffee of the world and about $99 \%$ of Latin American production.

Traditionally the propagation of this species is made through cuttings and seeds, both of these methods of propagation have disadvantages and limitations in production of clones in large number (Ismail et al., 2003). Seed propagation involves different problems such as, low germination rate, slow initial growth and short life span while vegetative cuttings guarantees uniformity seeds (Monaco et al., 1995; Ismail et al., 2003).

In vitro cultivation has relevance in the clonal propagation of valuable or endangered plant germplasm, and in the production of transgenic plants (Johnson and Emino, 1979; Smith et al., 1991; Frederic et al., 2007). To date, micropropagation techniques are employed to produce a large number of new and true-to-type plants in a relatively short period. It has already proved to be successful for several members of the Rubiacea family (Pierson et al., 1983; Carneiro and Ribeiro, 1989; Haidar, 1993; Ismail et al., 2003; Ebrahim et al., 2007; Almeasary, 2008). However, genotypic difference in shoot forming capacity in relation to the cytokinin level in the medium is not well documented.

The main aim of the present study is to find an efficient and simple method of in vitro clonal propagation using shoot tip explant for producing large numbers of Yemeni coffee cultivars, Coffea arabica cvs 'Benan', 'Burai', 'Odayni' and 'Odayni- Bayat'.

\section{MATERIALS AND METHODS}

Plant material and seed germination

Seeds: Healthy 12 years old coffee (C. arabica) trees grown in Yemen Republic were used as a source for seeds. Fruits of the four, Coffea arabica cvs 'Benan', 'Burai', 'Odayni' and 'Odayni-Bayat' were harvested in October, 2013. Seeds were obtained from mature and ripen fruits. The fruit crusts were separated from the pulp and seeds were washed with running water to remove the article horny. Seeds were then air dried under shade until having approximately $13 \%$ moisture content, which is suitable for storage.

The experiments were conducted on March $1^{\text {st }}$ 2014 until May1 $^{\text {st }} 2015$ at the Tissue Culture Lab, Department of Horticulture, Faculty of Agriculture, Suez Canal University, Ismailia - Egypt. The Coffea arabica cvs 'Benan', 'Burai', 'Odayni' and 'Odayni-Bayat' were used in this study.

\section{Surface sterilization}

Seeds were soaked in sterilized tap water for 24 hours, surface sterilization went through rinsing seeds with $70 \%$ ethanol solution for $30 \mathrm{~s}$, and then were rinsed in $20 \%(\mathrm{v} / \mathrm{v})$ sodium hypochlorite solution for $25 \mathrm{~min}$. The seeds were then washed three times with sterile tap water under laminar air-flow hood to remove all the traces of sodium hypochlorite.

Sterilized seeds were cultured into $40 \mathrm{ml}$ capacity jars containing $10 \mathrm{ml}$ quarter strength MS (Murashige and Skoog, 1962) basic salts and vitamins, 
supplemented with $20 \%(\mathrm{w} / \mathrm{v})$ sucrose, $7 \mathrm{gl}^{-1}$ agar, 1.0 $\mathrm{gl}^{-1}$ charcoal and the $\mathrm{pH}$ was adjusted to be 5.7 before autoclaving at $121^{\circ} \mathrm{C}$ and $1.2-1.3 \mathrm{~kg} / \mathrm{cm}^{2}$ pressure for $20 \mathrm{~min}$. One hundred seeds were cultured, one seed per jar. The cultures were incubated under growth room conditions $\left(22 \pm 2{ }^{\circ} \mathrm{C}\right.$, at dark). After germination, the seedlings were transferred into $16 \mathrm{~h}$ photoperiod with a light intensity of 4000 lux provided by florescent lamps (Phillips TLM 40W/33RS). The rate of germination was determined after 8 weeks from seed culture.

\section{Effects of BA on shoot induction}

After 12 weeks of seedling growth, shoot tips with the height of $(0.5 \mathrm{~cm})$ from in vitro culture explants were cultured onto $200 \mathrm{ml}$ capacity jars containing 40 $\mathrm{ml}$ MS medium supplemented with 3\% (w/v) sucrose, $7.0 \mathrm{gl}^{-1}$ agar and different concentrations of benzyl adenine (BA) viz, $\left(0.0,1.0,2.0,4.0,6.0\right.$ and $\left.8.0 \mathrm{mgl}^{-1}\right)$. Medium was adjusted to $\mathrm{pH} 5.7$ prior to autoclaving at $121^{\circ} \mathrm{C}$ and $1.2-1.3 \mathrm{~kg} / \mathrm{cm}^{3}$ pressure for $20 \mathrm{~min}$. Every jar contained one explant and each treatment had ten replicates. The cultures were grown for three months before data recording based on number of shoots, plant height, number of leaves/shoot clump as well as the culture fresh weight.

\section{Rooting of shoots}

For rooting, six-months-old in vitro shoots $(0.5$ $1.0 \mathrm{~cm}$ in length) were excised and transferred to $200 \mathrm{ml}$ capacity jars containing $40 \mathrm{ml}$ half strength MS medium supplemented with $15 \mathrm{gl}^{-1}$ sucrose, $7.0 \mathrm{gl}^{-1}$ agar and different concentrations of indole 3 -acetic acid (IAA), Indolebuteric acid (IBA) or NAA $(0.0,0.5,1.0,2.0$ and $3.0 \mathrm{mgl}^{-1}$ ) each. The cultures were placed under the same previously mentioned growth room conditions for root formation. After 8 weeks of culture, data were recorded based on the number of roots, root length, plant fresh weight and plant height.

\section{Acclimatization of plantlets}

The rooted shoots were carefully removed from the culture jars, then washed with running tap water to remove residual agar around the roots. Followed by washing with fungicide (Rizolex 1\%) to reduce the fungal contamination. The plantlets were transferred to a greenhouse for acclimation in plastic tray (209 cells) containing with a moist mixture of $(1: 1)$ sand and peat moss, then, maintained inside a plant growth chamber and irrigated with a fine mist of water for 3 weeks. The percentage of survived plants was determined after 4 weeks.

\section{Statistical analysis}

Experiments were set up in completely randomized design. Data were statistically analyzed using ANOVAIMANOVA of Statistica 6 software (Statsoft, 2001). The significance of differences among means was carried out using the Least Significant Test (L.S.D) at $=$ p 0.05 .

\section{RESULTS AND DISCUSSION}

\section{In vitro germination:}

Data in Figs. (1 and 2) showed that using quarter MS medium with and without activated charcoal was able to induce germination in coffee seeds for the four cultivars. Modified quarter MS medium with $1.0 \mathrm{~g} \mathrm{l}^{-1}$ activated charcoal gave the highest germination percentage $(85-87 \%)$ as compared to $70-75 \%$ without charcoal. of the four cultivars under study. The choice of medium that affect the germination, as has been reported for coffee plant and other species (Chen et al., 2015; Ebrahim et al., 2007).

\section{3-2. Shoot multiplication}

Explants were directly capable of developing multiple shoots on MS basal medium containing different concentrations of BA. Data in Table (1) and Fig. (2) showed that BA with the different concentrations, significantly increased the shoot number per explant compared to control treatment in all coffee cultivars under study. BA at the highest concentration $\left(8.0 \mathrm{mgl}^{-1}\right)$ gave the highest number of shoots, shoot length, number of leaves and fresh weight of shoot per explant (14.6, 2.64, 127.2 and 0.92), respectively, compared to control in case of Coffea arabica cv 'Benan' cultivar (Table 1 and Fig. 2 D). BA at 2.0, 4.0 and $6.0 \mathrm{mgl}^{-1}$ increased multiplication parameters significantly as compared to other BA concentrations in the case of Coffea arabica cvs 'Odayni', 'Burai' and 'Odayni-Bayat' respectively, with increment of BA concentration, the number of shoot per explant decreased in the three cultivars (Table 1). From this experiment, it was evident that best clump fresh weigh was obtained from MS medium amended with BA (2.0, 4.0 and $8.0 \mathrm{mgl}^{-1}$ ) which produced $0.56,0.64$ and $0.57 \mathrm{~g}$ fresh weight of shoots/explant from Coffea arabica cvs 'Odayni', 'Burai' and 'Odayni-Bayat', respectively. The positive effect of BA on the potential to induce plant regeneration in coffee plants has been reported previously by (Kahia and Owuor, 1990; Haidar, 1993; Ebrahim et al., 2007; Almeasary, 2008) who used BA in combination with different concentrations of auxin to induce in vitro multiplication in other coffee species. Comparing the present results of this species with the previous studies, it was seen that each species needs appropriate culture medium, with appropriate concentration of growth regulator.

\section{Root formation}

Data presented in Table (2) show the effect of IAA, IBA and/or NAA on the in vitro rooting of proliferated shoots of the four Coffea arabica cvs 'Benan', 'Burai', 'Odayni' and 'Odayni-Bayat.'. Root formation was different according to cultivars in response to the application of IAA, IBA and/or NAA to the culture media. The MS basic medium without IAA, IBA and/or NAA also revealed root formation. MS medium supplemented with the three kinds of auxins induced callus on the rooting stage in all the studied cultivars except at the tested concentrations of IBA and IAA in the case of Coffea arabica cv 'Odayni' (Fig. 2 E). The MS medium with NAA (2.0, 1.0 and $3.0 \mathrm{mgl}-1$ ) provides the highest number of roots per explant (3.39, 3.35 and 3.40 roots/explants) in Coffea arabica cv 'Odayni', 'Burai' and 'Odayni-Bayat' respectively, (Table 2 and Fig. 2 E). However, the highest number of roots per plantlet (3.44) was recorded with IBA $\left(1.0 \mathrm{mgl}^{-1}\right)$ in the case of Benan cultivar. Further increase in the concentrations of NAA and IAA had no effects on the 


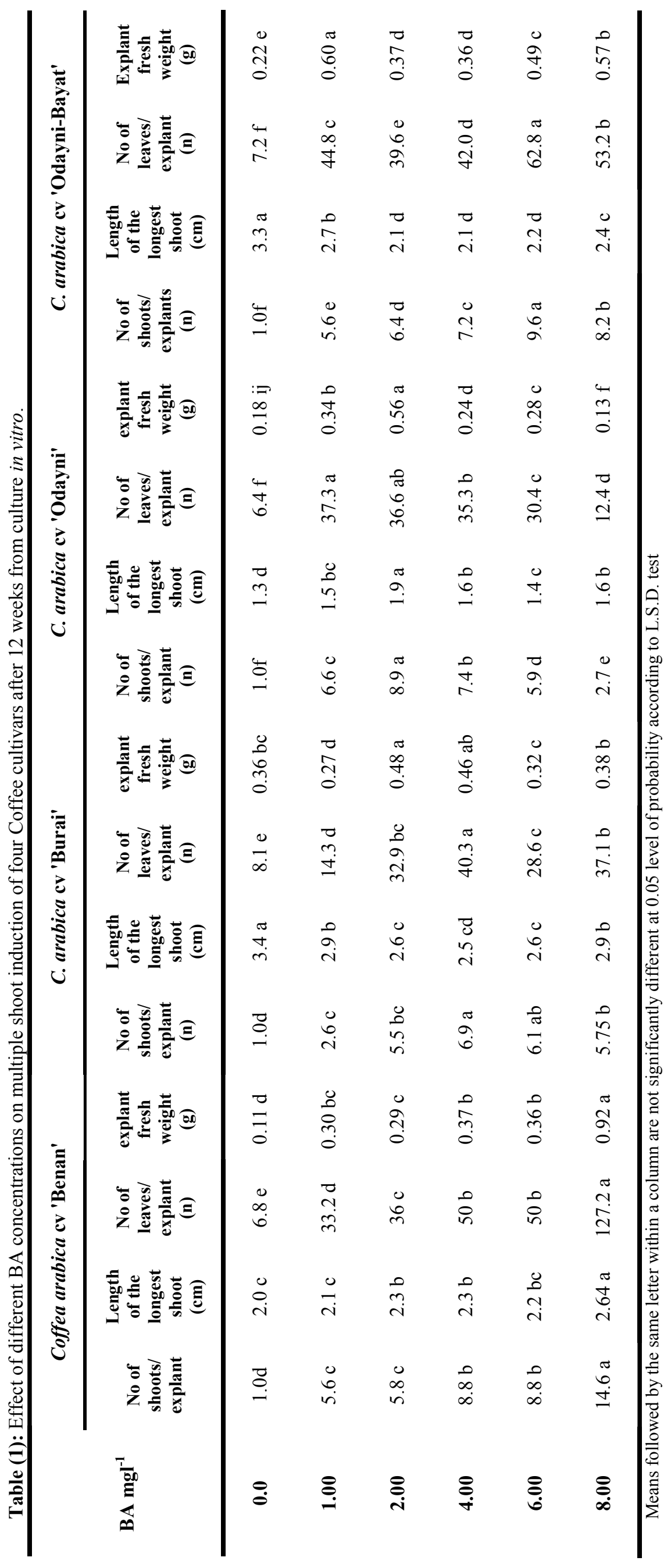




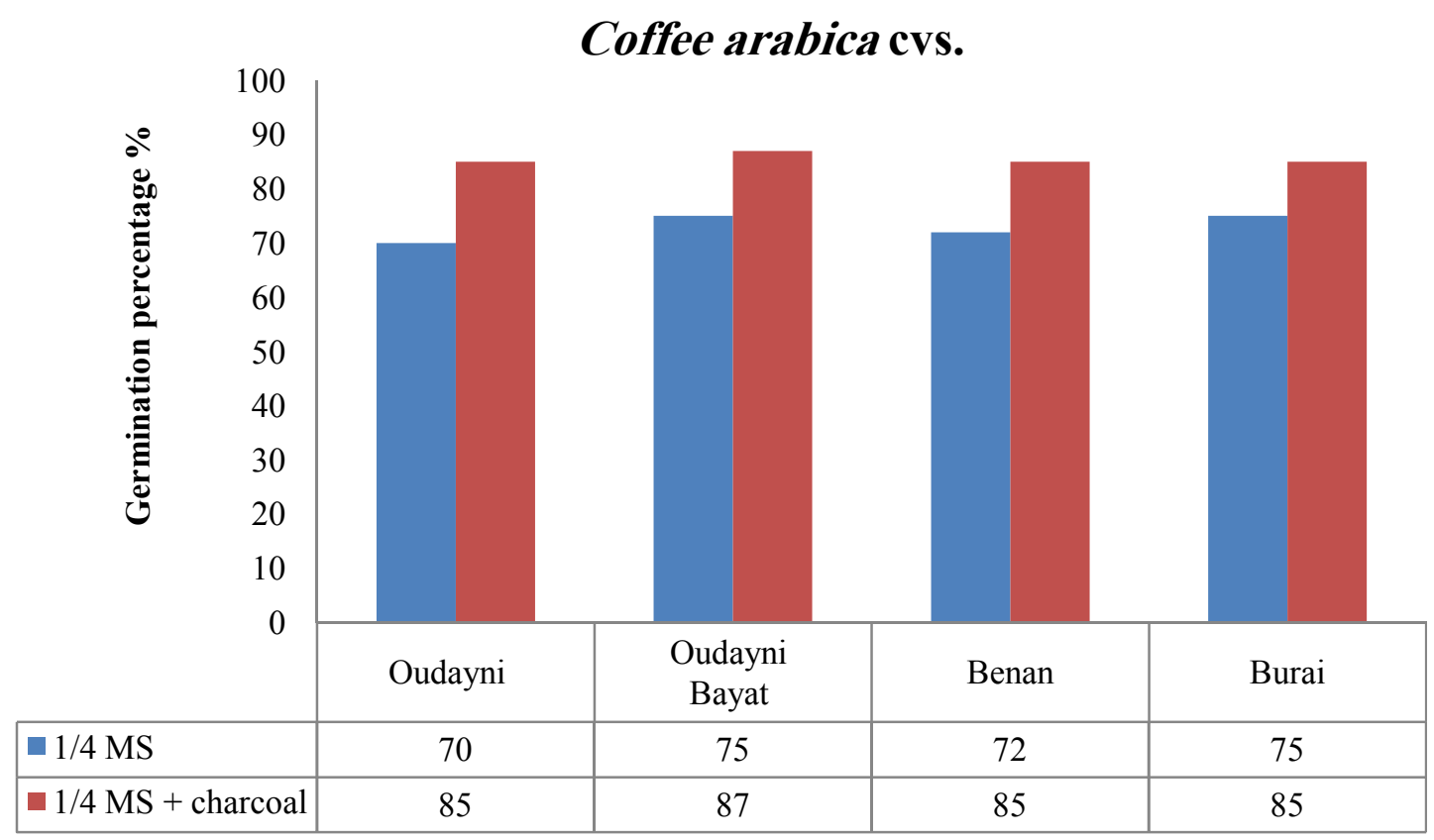

Fig. (1): Influence of different types of medium on germination of four Yemeni Coffea arabica cultivars.
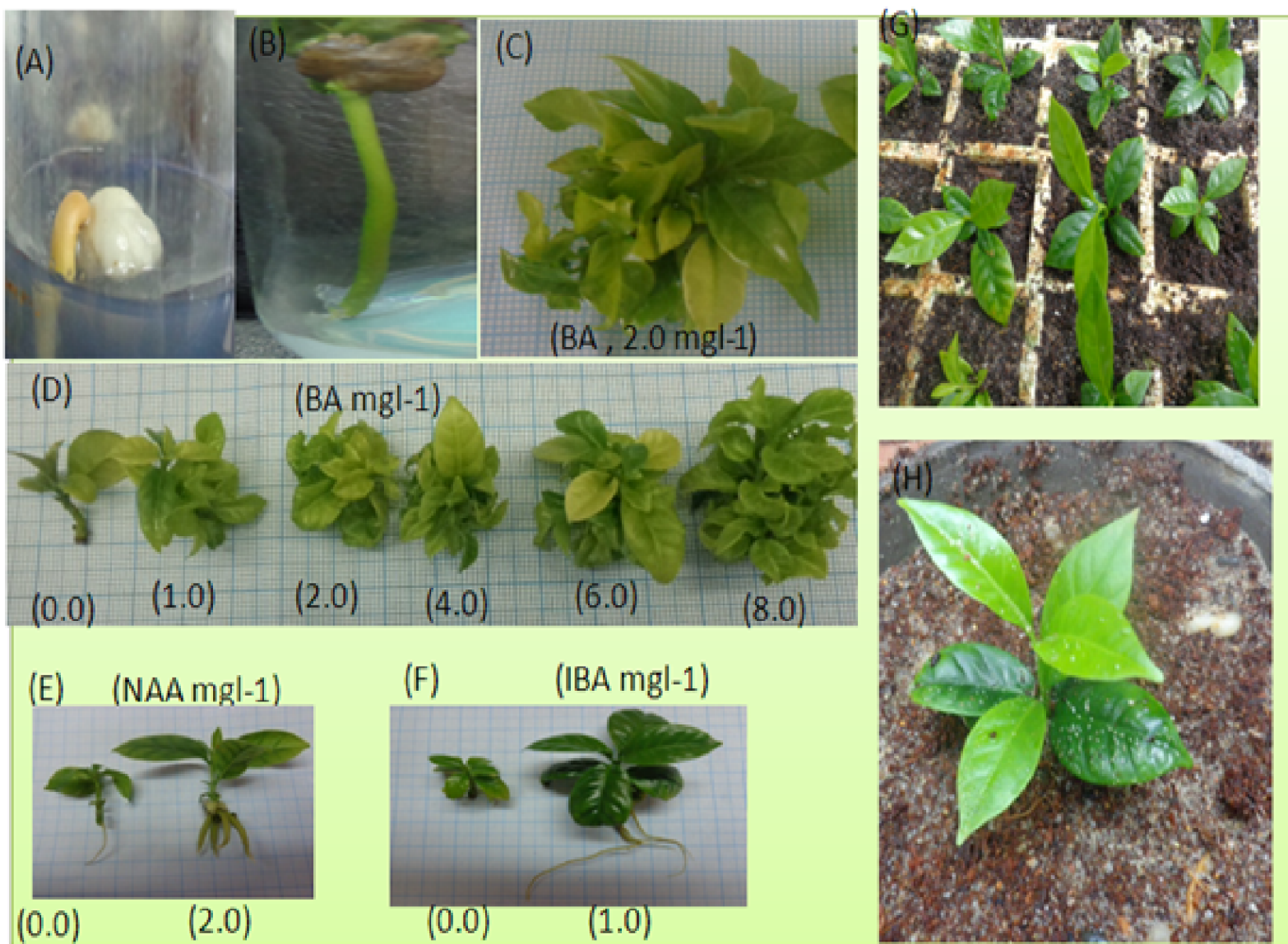

Fig. (2): Modified micropropagation method of Coffea arabica cultivars (A and B) germinated seed of $C$. arabica cv 'Oudayni - Bayat'. (C and D) multiple shoots generated from shoot tip explant of C. arabica cvs 'Oudayni' and 'Benan', respectively. (E) root formation of C. arabica cv 'Oudayni'. (F) root formation of $C$. arabica cv 'Benan'. (G and $\mathrm{H}$ ) plants of $C$. arabica cvs 'Burai' and 'Benan' in greenhouse after 4 and 8 weeks of acclimatization, respectively. 


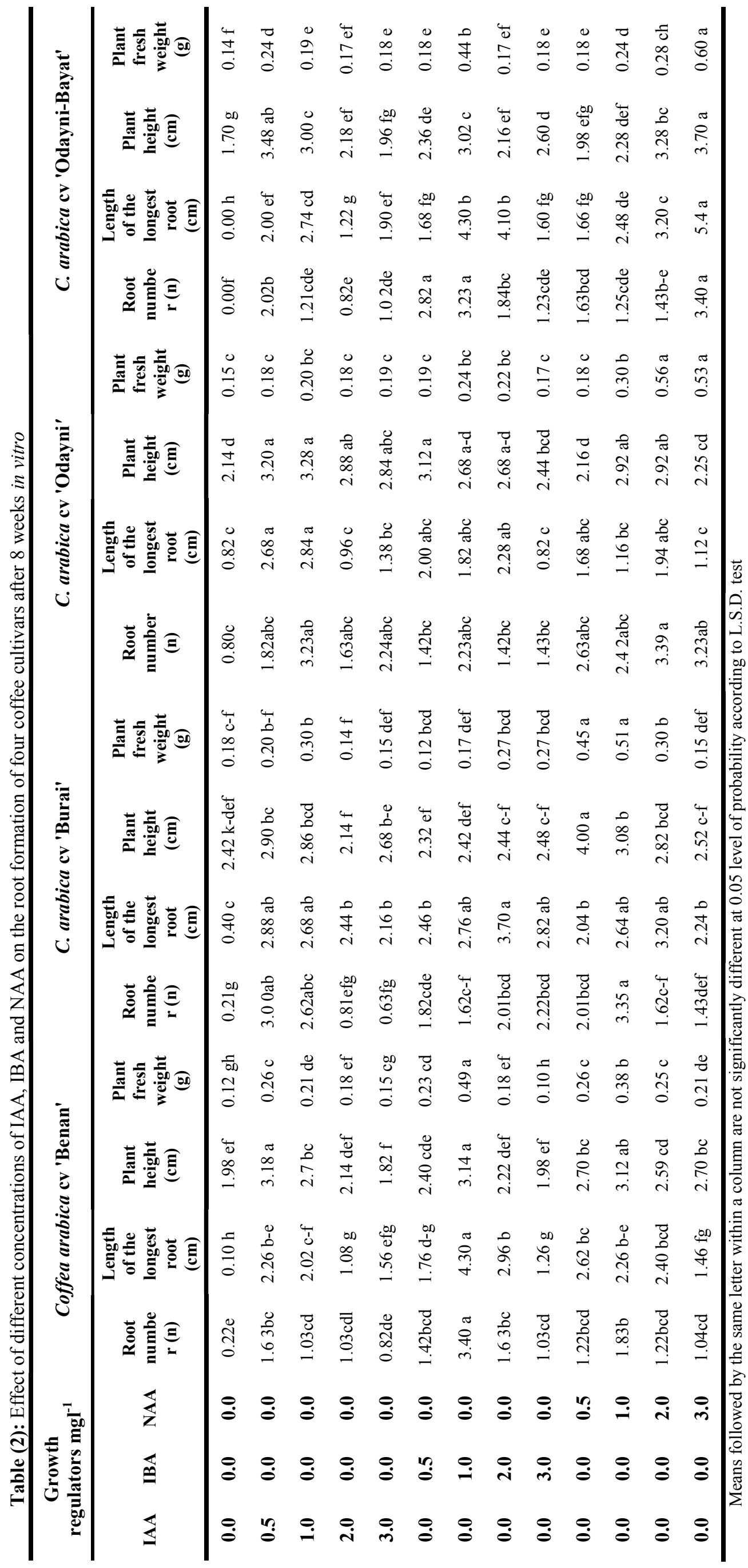


number of roots in the case of Coffea arabica cvs 'Odayni', 'Burai' and' Benan' (Table 2). The maximum root growth was recorded on MS medium with $1.0 \mathrm{mgl}^{-1}$ IBA, $2.0 \mathrm{mgl}^{-1}$ IBA, $1.0 \mathrm{mgl}^{-1}$ IAA and $3.0 \mathrm{mgl}^{-1} \mathrm{NAA}$ in the case of Coffea arabica cvs 'Benan' 'Burai', 'Odayni' 'Odayni-Bayat', respectively. Although excessive auxin in the medium is commonly characterized by callus formation, no callus formation was detected in rooting stage of all concentrations of IBA and IAA in the case of Coffea arabica cv 'Benan' (Fig. 2 F). The absence of callus at shoot base is an important observation because it can be excluded that auxin treatments were supplied in improper high supplements (Nerman et al., 2009). The obtained results are similar to the previous studies reported on many species by (Wamatu, 1990; Kahia and Owuor, 1990; Ganesh and Sreenath, 1997; Tiwari et al., 1999; khattab, 2011).

\section{Acclimatization}

The success of any in vitro micropropagation protocol largely depends on the survival and growth performance of the propagated plantlets ex-vitro (Joshi and Dhar, 2003). In the present study, the acclimation procedures applied was successful using the previously mentioned experimental conditions. Almost $90 \%$ of the regenerated plants of the four cultivars survived and showed vigorous growth (Fig. $2 \mathrm{G}$ and $\mathrm{H}$ ). In vitro derived plants did not display any phenotypic variation during subsequent vegetative development.

\section{ACKNOWLEDGMENTS}

The authors would like to thank Dr. Salah Khattab Department of Horticulture, Faculty of Agriculture, Suez Canal University for his assistance. All thanks to all staff member of Tissue Culture Lab, Suez Canal University, Ismailia, Egypt.

\section{REFERENCES}

Almeasary, M.A.S. (2008). Micropropagation in Coffee (Coffea arabica L). Ph.D. Dissertation, Faculty of Agriculture, Assiut university.

Carneiro, M.F. and T.M.O. Ribeiro (1989). In vitro micropropagation of Coffea arabica L. cv Caturra by means of axillary buds. Broteria Genetica, 85: 139-152.

Carneiro, M.F. (1997). Coffee biotechnology and its application in genetic transformation. Euphatica, 97: 167-172.

Carvalho, A. and L.C. Monaco (1969). The breeding of Arabica Coffee. In: Ferwerda, F.P. and F. Wit (eds.). Outlines of Perennial Crop Breeding in the Tropics, Veenman and Zonen NV., Wageningen, pp. 198-216.

Chen,Y., M.G. Uromi, Fan Xu-Li and G. Jiang-Y. (2015). Asymbiotic seed germination and in vitro seedling development of Paphiopedilum spicerianum: An orchid with an extremely small population in China. Global Ecology and Conservation, 3: 367-378.

Ebrahim, N., R. Shibli, I. Makhadmeh, M. Shatnawi and A. Abu-Ein (2007). In vitro Propagation and In vivo acclimatization of three coffee cultivars (Coffea arabica L.) from Yemen. World Applied Sciences Journal, 2(2): 142-150.

Frederic, N., D. Yingshan and L. Bao (2007). Somaclonal variation at the nucleotide sequence level in rice (Oryza sativa L.) as revealed by RAPD and ISSR markers, and by pairwise sequence analysis. J. Appl. Genet., 48: 329-336.

Ganesh, D.S. and H.L. Sreenath (1997). Clonal propagation of coffee through apical bud culture, J. Plant. Crops, 25: 169-174.

Haidar, A.K.A. (1993). Studies on the micropropagation of Coffea arabica L. M.Sc. Thesis, Zagazig University, Egypt.

Ismail, S., B. Naqvi, N. Anwar and R. Zuberi (2003). In vitro multiplication of Coffea arabica. Pak. J. Bot., 35(5): 829-834.

Johnson, J.L. and E. R. Emino (1979). In vitro propagation of Mammillariae longata. HortScience, 14: 605-606.

Joshi, M. and U. Dhar (2003). In vitro propagation of Saussurea obvallata (DC.) Edgew, an endangered ethnoreligious medicinal herb of Himalaya. Plant Cell Rep., 21: 933-939.

Juma, C., J. M. Magmbo and H. Monteith (1994). Tissue culture for coffee: The case of Uganda. Biotechnology and Development Monitor, 20: 19-20.

Kahia, J.W. and J.B.O. Owuor (1990). In vitro propagation of the disease resistant Coffea arabica L. Cultivar-Ruiru 11. Kenya-Coffee, 55: 901-905.

Khattab, S. (2011). Effect of Different Media and Growth Regulators on the in vitro Shoot Proliferation of Aspen, Hybrid Aspen and White Poplar Male Tree and Molecular Analysis of Variants in Micropropagated Plants. Life Science Journal, 8(1): 177-184.

Monaco, L.C., M.R. Sundahl, A. Carvalho, O.J. Crocomo and W.R. Sharp (1995). Application of tissue culture in the improvement of coffee. In: Reinert, J. and Y.P,S. Bajaj (Eds). Applied and Fundament Aspects of Plant Cell Tissue and Organ Culture. Narosa publ., India.

Murashige, T. and F. Skoog (1962). A revised medium for rapid growth and bioassays with tobacco tissue cultures. Physiol. Plant, 15: 473-497.

Nerman, M., E. Souad and T. Hussein (2009): In vitro mass propagation of the endangered Sainai Hawthorn Crataecus Sinaica boiss. International Journal of Academic Research, 1(1): 24-29.

Pierson, E.S., A.A.M. Van Lammeren, J.H.N. Schell and G. Startisky (1983). In vitro development of embryoids from punched leaf discs of Coffea canephora. Protoplasma, 115: 208-216.

Robinson, J. and B. Brian (1993). Coffee in Yemen, Practical guide .Technical Cooperation Federal Republic of Germany, GTZ -Yemen.

Smith, R.H., P.J. Burdick, J. Anthony and A.A. Reilley (1991). In Vitro Propagation of Coryphanthama cromeris. HortScience, 26(3): 315. 
Statsoft, Inc. (2001). STATISTICA for Windows (software-system fur Datenanalyse) Version 6. http: Iwww.statisoft.com.

Tiwari, K.P., S.K. Tiwari, M.C. Sharma and E.A. Siril (1999). Preliminary studies on micropropagation of Rubia cordifolia, a medicinal herb from central India.Vaniki- Sandesh, 23: 5-8.

Wamatu, J.N. (1990). Vegetative propagation of Ruiru11: a review. Kenya-Coffee., 55: 983-985.

\section{الإكثار الاقيق لأربعة أصناف من البن العربي اليمنية من خلال زراعة القمة النامية

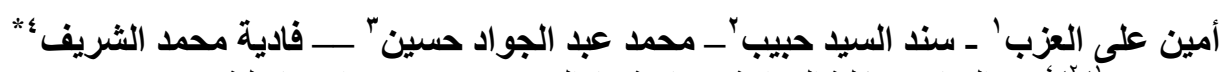

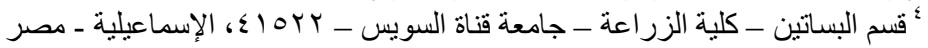

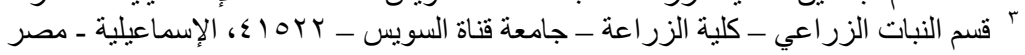

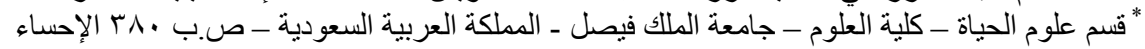

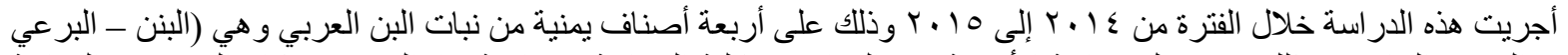

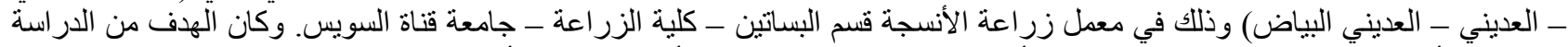

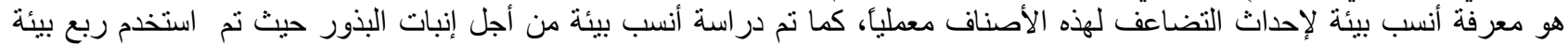

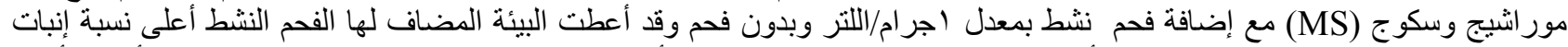

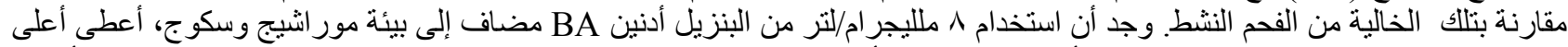

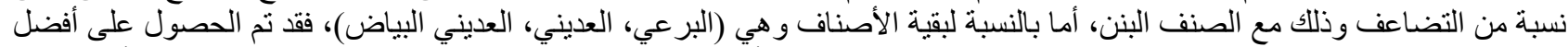

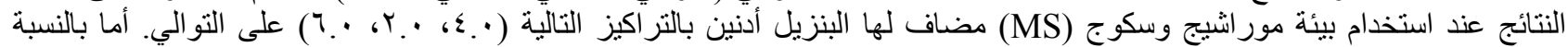

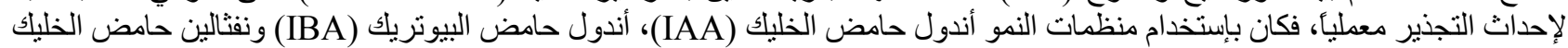
أعطت نسبة . • (NAA)

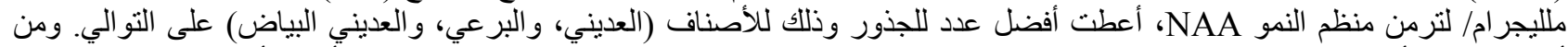

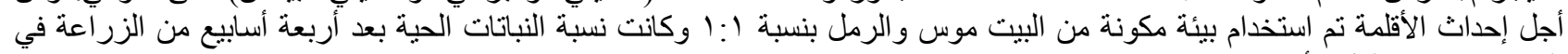

